\title{
Electrosmog and Its Protection
}

\author{
Manfred Doepp* \\ Holistic Center Head, Abtwil 9030, Switzerland \\ *Corresponding author: Manfred Doepp, Holistic Center, 13 Haupt St, Abtwil 9030, Switzerland. \\ To Cite This Article: Manfred Doepp. Electrosmog and Its Protection. Am J Biomed Sci \& Res. 2021 - 14(2). AJBSR.MS.ID.001971. \\ DOI: 10.34297/AJBSR.2021.14.001971.
}

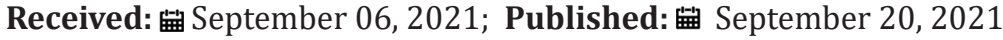

\section{Opinion}

There is hardly any doubt that our exposure to technical e-smog is increasing. Apart from the increasing natural-cosmicsolar irradiations, which are getting stronger and stronger, but which contain a meaning, because mankind is in a process of transformation, which is strengthened or guided by the cosmos. If you add up the current electromagnetic burdens, you come to a multiplication compared to what we already felt as an imposition 10 or even 20 years ago. And there is no improvement in sight, on the contrary: the more than 30,000 « $5 \mathrm{G} »$ or soon « $6 \mathrm{G} »$ satellites that are to be placed by the multi-billionaires (in future dollar trillionaires) are not yet in orbit. The "Internet of Things" is not supposed to make anything happen in an analogue way anymore (as nature does), but to link many billions of devices that are controlled from above and leave hardly any room for human decisions.

\section{Switching}

The optimisation of all functional processes is better achieved by computers and even artificial intelligence than by error-prone, irrational humans. Already in the January 2000 issue of the magazine Co 'Med, the author described that electrosmog (at that time « $2 \mathrm{G} »$ ) in the Salzburg area had a significant negative effect on meridian diagnostic results. A few years later, the author published that the phenomenon of brain "switching" was becoming more frequent, and he described exercises of deswitching (https://www. youtube.com/watch?v=0cvdRkCuFfA) to return paradoxical CNS reactions to the norm. Many teachers around the world use these exercises to bring their students, who can no longer live without a mobile phone and are therefore permanently switched, into a concentrated, learning state.

\section{Hertz (Schumann Frequency)}

In the meantime, all of this has multiplied or increased. Therefore, the question arises whether there are possibilities for protection against e-smog. There are many "chips" on the market that postulate this, and also larger devices. Undoubtedly, it makes sense to let the increasingly freak atmospheric mid-frequencies affect the body by means of small devices that emit the analogue frequency of $8 \mathrm{~Hz}$ (the Schumann frequency (https://www. youtube.com/watch?v=BM4aML9rrQc). Our organisms have been tuned to this frequency for many thousands of years, just as our brain knows and uses this as the middle frequency. Every sensitive person feels it when the real frequencies deviate far from it, by means of inexplicable indisposition and changes in mood.

\section{Scalar Waves}

With regard to the negative effects of technical E-smog on the body, the following must be stated: the generally recognised Hertzian waves $=$ transverse waves are not the biologically significant ones. Nature uses the Tesla = longitudinal = scalar waves in an analogous form. They are used militarily but are not officially recognised. Konstantin Meyl (www.kmeyl.de) has been trying to establish them for decades, but the resistance is great (https:// www.youtube.com/watch?v=nWfI5hYvE88). Unfortunately, there are no measuring instruments for scalar waves on the market so far, which leaves humans as the measuring instrument.

If one now demands of an e-smog protection that it should reduce the Hertzian waves, one will fail. This is because every mobile phone and every transmission mast will compensate 
for a drop in transmission intensity and increase the intensity, a counterproductive process. Protective devices that absorb or shield Hertz waves are therefore pointless. The primary readable reduction in the measuring device is a pseudo effect without positive effects on the organism. Therefore, Faraday cages as well as aluminium caps or wallpapers are not indicated. Longitudinal (oscillating in the direction of propagation) waves pass through them. Transversely oscillating transverse waves are stopped.

\section{Near Fields and The Brain}

Every emission of Hertzian waves by a transmitter (including mobile phones) creates an accumulation of chaotic, vagabond scalar vortices in its environment/near field. These spread out as equally chaotic scalar waves and are the main factor for biological stress. https://en.wikipedia.org/wiki/Zenneck_wave, https:// en.wikipedia.org/wiki/Longitudinal_wave, https://en.wikipedia. org/wiki/Near_and_far_field). Near-field and far-field of a transmitter: if the near-field vortices remained there, there would be no problem. However, they spread unchecked. The exposure when holding a mobile phone to the ear is extremely high.

\section{Thermal Effects?}

There is always a discussion whether the heating of the head is the essential criterion for exposure to a mobile phone. However, this thermal effect is only one piece of the mosaic; much more important is the disinformation of the cells as a result of the unnatural frequency patterns. The development of brain tumours increases strongly as a result, as does the above-mentioned phenomenon of switching, up to and including electrosmog addiction. The same protective principle applies as in all radiation medicine: the best radiation protection is to keep your distance. The most important button is the «off » button. And: fully electrified cars are highly problematic from this point of view. Scalar waves cannot be absorbed by electrically conductive materials, whereas dielectrics can. The effect of e-smog radiation as well as of protection can currently only be assessed on the basis of the reactions of autonomously regulated body systems, i.e. heart rate variability $=\mathrm{HRV}, \mathrm{EEG}$, skin resistance measurements esp. in acupuncture points, etc.

\section{Protection Methods}

If one divides the principles on which protective devices are based, they can essentially be subdivided:
1. Dielectrics informed against e-smog, mostly plastic chips.

2. Light metals, which are informed to harmonise e-smog.

3. Mineral mixtures of e.g., crystals, silicates, semi-precious stones.

According to the author's experience as results of many measurements and tests, the principles

a) and b) Work well at first, but they are subject to an informational-energetic erosion process. This means that in the course of months and at the latest years they lose their information density and have less and less effect on the body, after a longer period of time - depending on the intensity of the e-smog to be reduced - no effect at all. On the other hand, it is an advantage of principle.

b) That no information density is responsible, but the effect is based on the physical semiconductor and piezo properties. And these remain unchanged. If technical digital scalar waves penetrate such a crystal, it produces and emits analogue frequency-identical waves. Both types of waves overlap and stationary standing waves are created, which can then hardly affect the body. In addition, there is indeed a positive spiritual component due to the highly vibrating mineral crystals. Accordingly, the author could not detect any loss of effect over time with type

c) Devices, undoubtedly an advantage.

\section{Summary}

$5 \mathrm{G}$ is now a reality and will be rolled out everywhere, resistance is futile. «Industry $4.0 »$ with its total automation requires seamless and high-intensity coverage. Satellites are being launched into orbit for this purpose. As a result, many people suffer from considerable but seemingly inexplicable complaints and search in vain for a cause. Protection against or compensation for technical electrosmog has become an unavoidable necessity in our world. Man is and remains an analogue being of nature and will only become digital if he mutates into a transhuman being. Which could certainly be avoided. 